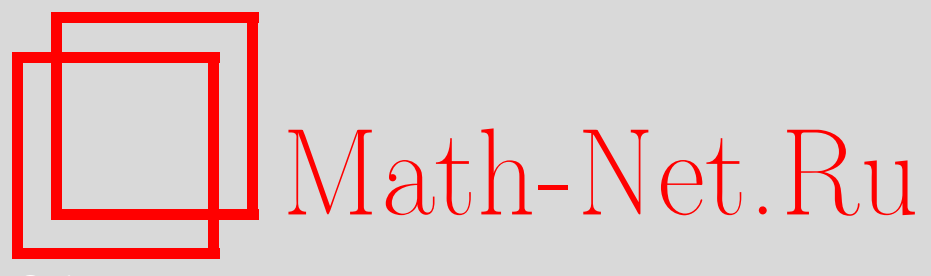

В. П. Громов, Задача Коши для уравнений в свертках в пространствах аналитических векторнозначных функций, Матем. заметки, 2007, том 82, выпуск 2, 190-200

DOI: https://doi.org/10.4213/mzm3798

Использование Общероссийского математического портала Math-Net.Ru подразумевает, что вы прочитали и согласны с пользовательским соглашением http://www . mathnet.ru/rus/agreement

Параметры загрузки:

IP : 18.207 .199 .55

26 апреля 2023 г., 14:44:38

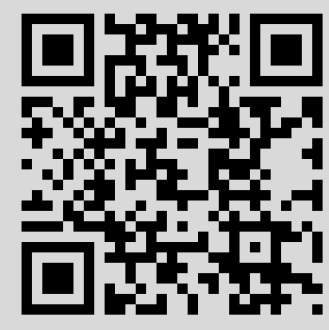


Том 82 выпуск 2 август 2007

УДК 517.55

\section{Задача Коши для уравнений в свертках в пространствах аналитических векторнозначных функций}

\section{В. П. Громов}

Данная работа посвящена задаче Коши неоднородных уравнений в свертках достаточно общей природы. Для решения поставленных задач применяется операторный метод, предложенный в более ранних работах автора. Решения рассматриваемых задач находятся конструктивно в форме хорошо сходящихся степенных векторнозначных рядов. Предлагаемый метод обеспечивает непрерывность получаемых решений относительно начальных данных и неоднородного члена уравнения.

Библиография: 10 названий.

\section{Введение}

Уравнения свертки широко распространены как в теоретических, так и в прикладных разделах современной математики. Они, как известно, содержат в себе многие классы дифференциальных и дифференциально-разностных уравнений и находят применение при разработке многих проблем естествознания, в частности квантовой теории физики (см. [1]). Полная теория таких уравнений далека от завершения.

Данная работа посвящена задаче Коши неоднородных уравнений в свертках достаточно общей природы. Для решения поставленных задач применяется операторный метод, предложенный в работах автора [2], [3]. Решения рассматриваемых задач находятся конструктивно в форме хорошо сходящихся степенных векторнозначных рядов. Предлагаемый метод обеспечивает непрерывность получаемых решений относительно начальных данных и неоднородного члена уравнения.

Отметим, что уравнения в свертках в настоящее время довольно активно изучаются в различных направлениях. Достаточно обширная библиография таких задач дана, например, в работах Тревса [4]. В частности, в обстоятельной, сравнительно недавней работе Стейнберга [5] рассмотрена задача Коши для уравнений свертки в пространствах функций вещественной переменной, быстро убывающих на бесконечности. В этой работе используется классический метод Фурье и, опираясь на теорему Овсянникова [6], доказаны теоремы существования и единственности решений без явного построения этих решений. 


\section{1. Дифференциально-операторные уравнения в свертках}

Воспользуемся следующими стандартными обозначениями: $z=\left(z_{1}, \ldots, z_{p}\right) \in \mathbb{C}^{p}$, $p \geqslant 1, z^{k}=z_{1}^{k_{1}} \cdots z_{p}^{k_{p}}, k_{p}$ - целые неотрицательные числа, $|k|=k_{1}+\cdots+k_{p}$, $(k)=\left(k_{1}, \ldots, k_{p}\right)$ - мультииндекс. Буквой $t$ в дальнейшем обозначается одномерная переменная: $t \in \mathbb{R}$ или $t \in \mathbb{C}$.

Пространство всех целых функций многих комплексных переменных с топологией равномерной сходимости на компактах обозначается $H\left(\mathbb{C}^{p}\right)$, пространство целых функций экспоненциального типа - $[1, \infty)$.

Рассмотрим уравнение

$$
\frac{d^{m} u(t, z)}{d t^{m}}=l_{0} * u(t, z)+F(z), \quad m \geqslant 1,
$$

где $l_{0}$ - фиксированный функционал, действующий по переменной $z, F(z)$ - фиксированная функция - неоднородный член уравнения.

Операция свертки, порождаемая функционалом $l_{0}$, порождает оператор $A$ по правилу

$$
A[u(t, z)]=l_{0} * u(t, z)=l_{0}[u(t, z+h)], \quad z+h=\left(z_{1}+h_{1}, \ldots, z_{p}+h_{p}\right) .
$$

В дальнейшем функционал $l_{0}$ и порождаемый им оператор $A$ уточняются.

Функцию

$$
\varphi_{0}(\xi)=l_{0}\left[e^{(\xi \cdot z)}\right], \quad \xi \cdot z=\left(\xi_{1} z_{1}+\cdots+\xi_{p} z_{p}\right)
$$

называем характеристической функиией оператора $A$. Если не оговорено противное, то в разделе 1 предполагается, что $l_{0} \in[1, \infty)^{*}$ и $\varphi_{0}(\xi)$ - целая функция многих комплексных переменных. Однако возможны и другие требования относительно $l_{0}$ и $\varphi_{0}(\xi)$.

Ставится следующая задача (задача Коши): найти решение $u(t, z)$ уравнения (1.1), удовлетворяющее начальным условиям:

$$
\left.\frac{d^{k} u(t, z)}{d t^{k}}\right|_{t=0}=f_{k}(z), \quad k=0,1, \ldots, m-1
$$

Имеет место

Теорема 1. Пусть $l_{0} \in[1, \infty)^{*}$. Задача (1.1), (1.2) имеет решение при любых заданных функииях $f_{0}, f_{1}, \ldots, f_{m-1}, F \in[1, \infty)$, и оно представляется в виде

$$
u(t, z)=\sum_{k=0}^{m-1} u_{k}\left(t, z, f_{k}\right)+u_{r}(t, z, F),
$$

¿əe

$$
\begin{aligned}
& u_{k}\left(t, z, f_{k}\right)=\sum_{n=0}^{\infty} \frac{A^{n}\left(f_{k}\right)(z)}{(n m+k) !} t^{n m+k}, \\
& u_{r}(t, z, F)=\sum_{n=0}^{\infty} \frac{A^{n}(F)(z)}{(n m+m) !} t^{m(n+1)} .
\end{aligned}
$$


Степенные ряды (1.4), (1.5) сходятся абсолютно по топологии пространства $H\left(\mathbb{C}^{p}\right)$ в каждой точке $t$ и представляют целье вектор-функиии экспоненциального типа. Решение (1.3) непрерывно относительно начальных данных и неоднородного члена уравнения.

Теорема 1 является одним из частных случаев более общего результата, относящегося к уравнениям в обобщенных производных Гельфонда-Леонтьева, к формулировке и доказательству которого мы и переходим.

Пусть дано уравнение

$$
D_{f}^{m} u(t, z)=l_{0} * u(t, z)+F(z), \quad m \geqslant 1,
$$

где правая часть уравнения такая же, как в (1.1), а слева - обобщенная производная Гельфонда-Леонтьева порядка $m \geqslant 1$, действующая по переменной $t \in \mathbb{C}$, порожденная целой функцией $f$ порядка роста $\rho>0$ конечного типа $\sigma>0$ и удовлетворяющая условию регулярности

$$
f(z)=\sum_{n=0}^{\infty} a_{n} z^{n}, \quad a_{n} \neq 0, \quad \lim _{n \rightarrow \infty} n^{1 / \rho} \sqrt[n]{\left|a_{n}\right|}=(\sigma e \rho)^{1 / \rho} .
$$

Не ограничивая общности, полагаем $a_{0}=1$.

Операторы $D_{f}^{m}$ введены в совместной работе [1] Гельфонда и Леонтьева и широко используются в различных задачах анализа. Отметим, в частности, что если $f(z)=e^{z}$, то $D_{f}^{m}=d^{m} / d t^{m}$. Так что уравнение $(1.1)$ - действительно частный случай уравнения (1.6).

Ставится задача (задача Коши): найти решение уравнения (1.6), удовлетворяющее следующим начальным условиям:

$$
\left.D_{f}^{m} u(t, z)\right|_{t=0}=f_{k}(z), \quad k=0,1, \ldots, m-1 .
$$

\section{Справедлива}

Теорема 2. Пусть $l_{0} \in[1, \infty)^{*}$. Задача (1.6), (1.7) имеет решение при любъх заданных начальных функииях $f_{0}, f_{1}, \ldots, f_{m-1} \in[1, \infty)$ и любом $F \in[1, \infty)$, и это решение представляется суммой вида (1.3), где

$$
\begin{aligned}
& u_{k}\left(t, z, f_{k}\right)=\sum_{n=0}^{\infty} a_{n m+k} A^{n}\left(f_{k}\right)(z) t^{n m+k}, \\
& u_{r}(t, z, F)=\sum_{n=0}^{\infty} a_{m(n+1)} A^{n}(F)(z) t^{m(n+1)} .
\end{aligned}
$$

Ряды (1.8), (1.9) сходятся абсолютно по топологии пространства $H\left(\mathbb{C}^{p}\right)$ в каждой точке $t \in \mathbb{C}$ и представляют целые вектор-функиии, порядок роста которых не превосходит $\rho$. Решение (1.3) непрерывно относительно начальных данных и неоднородного члена уравнения.

Напомним, что здесь $\left\{a_{n}\right\}$ - тейлоровские коэффициенты фиксированной функции $f$, порождающей операторы $D_{f}^{m}$, а $\rho$ - ее порядок роста, $A$ - оператор свертки, порожденный функционалом $l_{0}$. 
ДокАЗАтЕЛЬСтво теоремы 2. Оператор свертки $A(\Phi)=l_{0} * \Phi(z)$ определим на пространстве $[1, \infty)$ целых функций экспоненциального типа. Именно этому пространству принадлежат начальные данные и неоднородный член уравнения (1.6). Этот оператор непрерывно действует на $[1, \infty)$, переводит пространство $[1, \infty)$ в себя и имеет порядок $\beta(A)=0$ и тип $\alpha(A)=\infty$. Действительно, каждая функция $\Phi \in[1, \infty)$ представляется следующим интегралом Бореля:

$$
\Phi(z)=\frac{1}{(2 \pi i)^{p}} \int_{\Delta r+\varepsilon} e^{(\xi \cdot z)} \gamma_{p}(\xi) d \xi, \quad \xi \cdot z=\left(\xi_{1} z_{1}+\cdots+\xi_{p} z_{p}\right),
$$

где $\gamma_{p}(\xi)$ - функция, ассоциированная по Борелю с функцией $\Phi, \Delta r+\varepsilon-$ тор $\left|\xi_{i}\right|=r_{i}$, $i=1, \ldots, p,\left\{r_{i}\right\}$ - сопряженные радиусы функции $\gamma_{p}(\xi), \varepsilon>0$.

Действуя оператором $A^{n}$ на функции $\Phi(z)$, находим

$$
A^{n}(\Phi)(z)=\frac{1}{(2 \pi i)^{p}} \int_{\Delta r+\varepsilon} e^{(\xi \cdot z)} \gamma_{p}(\xi) \varphi^{n}(\xi) d \xi \in[1, \infty), \quad n=0,1, \ldots,
$$

где $\varphi_{0}(\xi)$ - характеристическая функция оператора $A$.

Далее, рассмотрим преобразование Фурье-Лапласа

$$
F(l)=l\left[e^{(\xi \cdot z)}\right]=\varphi(\xi), \quad \forall l \in[1, \infty)^{*} .
$$

Известно [1], что оператор $F$ осуществляет топологический изоморфизм

$$
[1, \infty)^{*} \approx H\left(\mathbb{C}^{p}\right)
$$

При этом оператор $A^{*}$, сопряженный с $A$, преобразуется в оператор умножения в пространстве $H\left(\mathbb{C}^{p}\right)$ на характеристическую функцию $\varphi_{0}(\xi)$ оператора $A$, т.е.

$$
A^{*} \approx B(\varphi)=\varphi_{0} \cdot \varphi \quad \forall \varphi \in H\left(\mathbb{C}^{p}\right) .
$$

Оператор умножения в $H\left(\mathbb{C}^{p}\right)$ имеет нулевой порядок и бесконечный тип, следовательно, $\beta\left(A^{*}\right)=0, \alpha\left(A^{*}\right)=\infty$. Пространство $[1, \infty)$ рефлексивно, а в рефлексивных пространствах справедливы равенства $\beta(A)=\beta\left(A^{*}\right), \alpha(A)=\alpha\left(A^{*}\right)$ (см. [8]), и поэтому $\beta(A)=0, \alpha(A)=\infty$.

Нулевой порядок оператора $A$ обеспечивает абсолютную сходимость рядов (1.8) и (1.9) и их непрерывную зависимость от функций $f_{0}, f_{1}, \ldots, f_{m-1}$ и $F$ соответственно.

Непосредственной подстановкой функций $u_{k}$ и $u_{r}$, опираясь на свойства оператора $D_{f}^{m}$, нетрудно убедиться, что

$$
D_{f}^{m}\left(u_{k}\right)=A\left(u_{k}\right), \quad k=0,1, \ldots, m-1,\left.\quad D_{f}^{s} u_{k}\left(t, z, f_{k}\right)\right|_{t=0}=\delta_{s k}, \quad 0 \leqslant s \leqslant m-1 .
$$

Легко проверяется также, что функция $u_{r}(t, z, F)$ удовлетворяет уравнению (1.6) и всем начальным условиям:

$$
D_{f}^{m} u_{r}(t, z, F)=A\left(u_{r}(t, z, F)\right)+F(z),\left.\quad D_{f}^{s} u_{r}(t, z, F)\right|_{t=0}=0, \quad 0 \leqslant s \leqslant m-1 .
$$

Следовательно, сумма (1.3), где функции $u_{k}$ и $u_{r}$ определяются соответственно рядами (1.8) и (1.9), являются решениями задачи (1.6), (1.7). Наконец, по формулам, определяющим характеристики роста степенного ряда по его коэффициентам, находим, что $u_{k}$ и $u_{r}$ - целые вектор-функции, порядок роста которых не превосходит $\rho$. Теорема доказана. 
Теорема 1, как отмечалось выше, является одним из частных случаев теоремы 2; она следует из теоремы 2 , если $f(z)=e^{z}$.

Выделим два наиболее часто встречающихся в практике случая: $m=1, m=2$. В этих случаях ряды, представляющие решения, суммируются, и решения представляются красивыми компактными формулами.

ПрЕДЛОЖЕНИЕ 1. Пусть $m=1$, тогда задача (1.6), (1.7) имеет решение при любых $f_{0}, F \in[1, \infty)$. Оно непрерьвно относительно функиий $f_{0}$ и $F$ и представляется формулой

$$
u(t, z)=\frac{1}{(2 \pi i)^{p}} \int_{\Delta r} e^{(\xi \cdot z)}\left\{f(t \varphi(\xi)) \gamma_{0}(\xi)+\left[\frac{f(t \varphi(\xi))-1}{\varphi(\xi)}\right] \gamma_{F}(\xi)\right\} d \xi,
$$

где $\gamma_{0}(\xi), \gamma_{p}(\xi)$ - функции, ассоциированные по Борелю с $f_{0} u F, \Delta r-$ тор $\left|\xi_{i}\right|=r_{i}$, $i=1, \ldots, p$, на котором функции $\gamma_{0}$ и $\gamma_{F}$ аналитические и на котором нет нулей

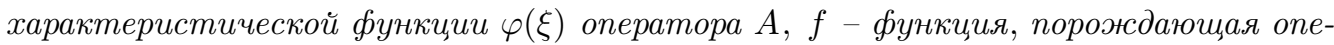
раторы $D_{f}^{m}, u(t, z)$ - челая вектор-функция со значениями в $[1, \infty)$ порядка роста, не превосходящего $\rho$.

ПРЕДЛОЖЕНИЕ 2. Пусть $m=2$, тогда задача (1.6), (1.7) имеет решение при любых $f_{0}, f_{1}, F \in[1, \infty)$, оно непрерывно относительно функциц $f_{0}, f_{1}, F$ и представляется следующей формулой:

$$
\begin{aligned}
& u(t, z)=\frac{1}{(2 \pi i)^{p}} \int_{\Delta r} e^{(\xi \cdot z)}\left\{\gamma_{0}(\xi) \operatorname{Kh}(t \sqrt{\varphi(\xi)})\right. \\
& \left.\quad+\gamma_{1}(\xi) \frac{\operatorname{Sh}(t \sqrt{\varphi(\xi)})}{\sqrt{\varphi(\xi)}}+\gamma_{F}(\xi)\left[\frac{\operatorname{Kh}(t \sqrt{\varphi(\xi)})-1}{\varphi(\xi)}\right]\right\} d \xi,
\end{aligned}
$$

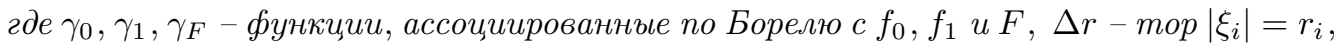
$i=1, \ldots, p$, на котором функции $\gamma_{0}, \gamma_{1}, \gamma_{F}$ аналитические и на котором нет нулей характеристической функиии $\varphi(\xi)$ оператора $A, \operatorname{Kh}(z)=[f(z)+f(-z)] \cdot 2^{-1}$, $\operatorname{Sh}(z)=[f(z)-f(-z)] \cdot 2^{-1}, u(t, z)$ - целая вектор-функиия со значениями в $[1, \infty)$ и ее порядок роста не превосходит $\rho$.

Заметим, что если $f(z)=e^{z}$, то $\operatorname{Kh}(z)$ и $\operatorname{Sh}(z)$ являются гиперболическими косинусом и синусом.

Предложения 1 и 2 являются следствием теоремы 2. Формулы (1.10) и (1.11) доказываются, исходя из представлений (1.8) и (1.9), используя преобразование Бореля функции пространства $[1, \infty)$. Действительно, каждая функция $\Phi \in[1, \infty)$ представляется интегралом Бореля:

$$
\Phi(z)=\frac{1}{(2 \pi i)^{p}} \int_{\Delta r+\varepsilon} e^{(\xi \cdot z)} \gamma_{\Phi}(\xi) d \xi, \quad(\xi \cdot z)=\left(\xi_{1} z_{1}+\cdots+\xi_{p} z_{p}\right),
$$

где $\gamma_{\Phi}(\xi)$ - функция, ассоциированная по Борелю с функцией $\Phi, \Delta r+\varepsilon-$ тор $\left|\xi_{i}\right|=$ $r_{i}, i=1, \ldots, p,\left\{r_{i}\right\}$ - сопряженные радиусы функции $\gamma_{p}(\xi), \varepsilon>0$.

Действуя на $\Phi(z)$ оператором $A^{n}$, находим

$$
A^{n}[\Phi](z)=\frac{1}{(2 \pi i)^{p}} \int_{\Delta r+\varepsilon} e^{(\xi \cdot z)} \gamma_{\Phi}(\xi) \varphi^{n}(\xi) d \xi, \quad n=0,1, \ldots,
$$

где $\varphi(\xi)$ - характеристическая функция оператора $A$. 
Подставляя эти выражения в равенства (1.8), (1.9) и (1.3) и суммируя соответствующие ряды при $m=1$ и $m=2$, получаем искомые формулы (1.10) и (1.11). Еще раз отметим, что если $f(z)=e^{z}$, то из формул (1.10) и (1.11) следуют компактные формулы, описывающие решения задачи (1.1), (1.2) при $m=1$ и $m=2$.

\section{2. Уравнения бесконечного порядка в обобщенных частных производных Гельфонда-Леонтьева}

В этом разделе результаты раздела 1 распространяются на дифференциально-операторные уравнения в обобщенных свертках, содержащие обобщенные частные производные Гельфонда-Леонтьева.

2.1. Как известно, операторы обобщенного дифференцирования Гельфонда-Леонтьева определены для аналитических функций одного переменного (см. [9]). Аналогичные операторы можно рассматривать и на пространствах функций многих комплексных переменных. Действительно, пусть

$$
\tilde{f}(z)=\sum_{(n)} a_{(n)} z^{n}, \quad z \in \mathbb{C}^{p}, \quad(n)=\left(n_{1}, \ldots, n_{p}\right),
$$

- фиксированная целая функция конечного порядка $\rho_{1}>0$ типа $\sigma \neq 0, \infty$, удовлетворяющая следующему условию:

$$
\forall a_{(n)} \neq 0 \quad \underline{\lim }_{|n| \rightarrow \infty}|n|^{1 / \rho_{1}} \sqrt[|n|]{\left|a_{(n)}\right|}>0
$$

Не ограничивая общности, полагаем $a_{(0)}=1$.

Пусть также $F(z)=\sum_{(n)} b_{(n)} z^{n}, z=z_{1}^{n_{1}} \cdots z_{p}^{n_{p}},-$ производная целая функция.

По определению выражение

$$
\begin{gathered}
D_{\tilde{f}}^{(m)}(F)(z)=\sum_{(n)} \frac{b_{(n+m)}}{a_{(n+m)}} a_{(n)} z^{n}, \\
(m)=\left(m_{1}, \ldots, m_{p}\right), \quad(n+m)=\left(n_{1}+m_{1}, \ldots, n_{p}+m_{p}\right)
\end{gathered}
$$

назовем обобщенной частной производной Гелъфонда-Леонтъева функции $F$ порядка $(m)$, порожденной функцией $f$.

В силу условия (2.1) ряды (2.2) сходятся абсолютно в каждой точке и представляют целую функцию $D_{\tilde{f}}^{(m)}(F)(z)$.

Отметим, что в данной работе операторы (2.2) используются и вводятся нами лишь в пространствах целых функций. Однако такие операторы можно рассматривать и в других пространствах функций многих комплексных переменных.

Приведем некоторые свойства оператора $D_{\tilde{f}}^{(m)}$, вытекающие из его определения:

1) $D^{(m)}\left[\alpha F_{1}+\beta F_{2}\right]=\alpha D^{(m)}\left(F_{1}\right)+\beta D^{(m)}\left(F_{2}\right)$ для всех $\alpha, \beta \in \mathbb{C}, F_{1}, F_{2} \in H\left(\mathbb{C}^{p}\right)$;

2) $D^{(m)}(C)=0, C=$ const;

3) $D^{(m)}\left[D^{(s)}(F)\right]=D^{(s)}\left[D^{(m)}(F)\right]=D^{(m+s)}(F), m+s=\left(m_{1}+s_{1}, \ldots, m_{p}+s_{p}\right)$;

4) $D_{\tilde{f}}^{(m)}[\tilde{f}(\xi \cdot z)]=\xi_{1}^{m_{1}} \cdots \xi_{p}^{m_{p}} \tilde{f}(\xi \cdot z)$ для всех $\xi, z \in \mathbb{C}^{p},(\xi \cdot z)=\left(\xi_{1} z_{1}, \ldots, \xi_{p} z_{p}\right)$; 
5) если $\tilde{f}(z)=e^{z_{1}} \cdots e^{z_{p}}$, то

$$
D_{\tilde{f}}^{(m)}=\frac{\partial^{m_{1}+\cdots+m_{p}}}{\partial z_{1}^{m_{1}} \cdots \partial z_{p}^{m_{p}}}
$$

Эти свойства показывают, что выражение (2.2) действительно можно трактовать как обобщение частных производных функций.

Используя формулу Коши, легко показать, что $D_{\tilde{f}}^{(m)}-$ непрерывный оператор на пространстве $H\left(\mathbb{C}^{p}\right)$, переводящий это пространство в себя.

2.2. Обобщенное преобразование Бореля. Пусть $F(z)=\sum_{(n)} b_{(n)} z^{n}-$ произвольная целая функция многих комплексных переменных, порядок роста которой не превосходит $\rho>0$ и конечного типа. Пространство всех таких функций в дальнейшем обозначаем $[\rho, \infty)$.

Каждой $F \in[\rho, \infty)$ поставим в соответствие функцию

$$
\gamma_{F}(\xi)=\sum_{(n)} \frac{b_{(n)}}{a_{(n)} \xi^{n+1}}, \quad n+1=\left(n_{1}+1, \ldots, n_{p}+1\right), \quad \xi^{n+1}=\xi_{1}^{n_{1}+1} \cdots \xi_{p}^{n_{p}+1}
$$

где $a_{(n)}$ - тейлоровские коэффициенты функции $f$ порядка $\rho$, удовлетворяющие условию (2.1).

В силу условия (2.1) ряд (2.3) сходится равномерно в некоторой области $G \subset \mathbb{C}^{p}$, содержащей бесконечно удаленную точку $\left(\xi_{1}=\cdots=\xi_{p}=\infty\right)$, и определяет функцию $\gamma_{F}(\xi)$, аналитическую в этой области. Функцию $(2.3)$ в дальнейшем называем функиией, обобщенно ассоциированной с $F(z)$, порожденной $f$.

Отметим, в частности, что если $f(z)=e^{z_{1}} \cdots e^{z_{p}}$, то (2.3) представляет классическую функцию, ассоциированную по Борелю с $F(z)$ (см. [10]).

Легко проверяется справедливость следующего интегрального представления:

$$
F(z)=\frac{1}{(2 \pi i)^{p}} \int_{\Delta r+\varepsilon} f(\xi \cdot z) \gamma_{F}(\xi) d \xi, \quad(\xi \cdot z)=\left(\xi_{1} z_{1}, \ldots, \xi_{p} z_{p}\right),
$$

где $\Delta r+\varepsilon-$ тор $\left|\xi_{i}\right|=r_{i}, \varepsilon>0, i=1, \ldots, p,\left\{r_{i}\right\}$ - сопряженные радиусы ряда (2.3) (см. [10]).

Теперь перейдем к уравнениям.

2.3. Рассмотрим уравнение

$$
D_{f}^{m} u(t, z)=\sum_{(k)} c_{(k)} D_{\tilde{f}}^{(k)}[u(t, z)]+F(z), \quad m \geqslant 1,
$$

где $D_{f}^{m}$ - одномерный оператор обобщенного дифференцирования порядка $m \geqslant 1$, порожденный функцией $f$ порядка роста $\rho>0$ и конечного типа, удовлетворяющей условию регулярности $(*)$ (см. раздел 1$)$, и действующий по переменной $t ; D_{\tilde{f}}^{(k)}-$ многомерный оператор обобщенной частной производной порядка $(k)$, порожденный целой функцией $\tilde{f}$ многих комплексных переменных порядка роста $\rho_{1}>0$, конечного типа с условием (2.1) и действующий по переменной $z \in \mathbb{C}^{p} ; F(z)$ - фиксированная функция - неоднородный член уравнения (2.5). 
Функция

$$
\varphi(\xi)=\sum_{(k)} c_{(k)} \xi^{k}, \quad \xi^{k}=\xi_{1}^{k_{1}} \cdots \xi_{p}^{k_{p}},
$$

называется характеристической функиией оператора

$$
A=\sum_{(k)} c_{(k)} D_{\tilde{f}}^{(k)}
$$

В дальнейшем предполагается, что $\varphi(\xi)$ - целая функция многих комплексных переменных.

Очевидно, что уравнение (2.5) является широким обобщением дифференциальных уравнений в частных производных бесконечного порядка, которые активно изучаются последние десятилетия. А выражение $\sum_{(k)} c_{(k)} D_{\tilde{f}}^{(k)} F$ - обобщенная свертка функции $F$ с некоторым функционалом.

Поставим задачу (задача Коши): найти решение $u(t, z)$ уравнения $(2.5)$, удовлетворяющее следующим начальным условиям:

$$
\left.D_{f}^{k} u(t, z)\right|_{t=0}=f_{k}(z), \quad k=0,1, \ldots, m-1 .
$$

Такую задачу решает

Теорема 3. Задача (2.5), (2.6) имеет решение при любых заданных (начальных) бункииях $f_{0}, f_{1}, \ldots, f_{m-1} \in\left[\rho_{1}, \infty\right)$ и любом неоднородном члене $F \in\left[\rho_{1}, \infty\right)$, и это решение представляется рядом вида

$$
u(t, z)=\sum_{k=0}^{m-1} u_{k}\left(t, z, f_{k}\right)+u_{r}(t, z, F)
$$

¿əe

$$
\begin{aligned}
& u_{k}\left(t, z, f_{k}\right)=\sum_{n=0}^{\infty}\left\{\frac{a_{n m+k}}{(2 \pi i)^{p}} \int_{\Delta r} \tilde{f}(\xi \cdot z) \gamma_{k}(\xi) \varphi^{n}(\xi) d \xi\right\} t^{n m+k} \\
& u_{r}(t, z, F)=\sum_{n=0}^{\infty}\left\{\frac{a_{n m+m}}{(2 \pi i)^{p}} \int_{\Delta r} \tilde{f}(\xi \cdot z) \gamma_{F}(\xi) \varphi^{n}(\xi) d \xi\right\} t^{m(n+1)}
\end{aligned}
$$

$\gamma_{0}, \ldots, \gamma_{m-1}, \gamma_{F}-$ обобщенные ассоциированные функции соответственно с функиией $f_{k}, k=0, \ldots, m-1, F, \Delta r+\varepsilon-\operatorname{mop}\left|\xi_{i}\right|=r_{i}, \varepsilon>0, i=1, \ldots, p$, на котором функции $\gamma_{k}, \gamma_{F}$ аналитические.

Рядъ (2.8), (2.9) сходятся абсолютно по топологии пространства в каждой точке $t \in \mathbb{C}$ и представляют целье вектор-функции со значениями в пространстве $[\rho, \infty)$, порядки роста которых не превосходят $\rho_{1}$. Решение $(2.7)$ непрерывно относительно начальных данных $f_{1}, \ldots, f_{m-1}$ и неоднородного члена $F$ уравнения (2.5).

ДокАЗАТЕЛЬСтво теоремы 3. Запишем уравнение (2.5) в виде

$$
D_{f}^{m} u(t, z)=A[u(t, z)]+F(z), \quad A=\sum_{(k)} c_{(k)} D_{\tilde{f}}^{(k)}
$$


Оператор $A$ определен на пространстве $\left[\rho_{1}, \infty\right)$ целых функций многих комплексных переменных порядка роста, не превосходящего $\rho_{1}\left(\rho_{1}-\right.$ порядок роста функции $f$ ).

Пользуясь обобщенным преобразованием Фурье с ядром $\tilde{f}$, находим, что порядок оператора $A$ равен $\beta(A)=0$ и его тип $\alpha(A)=\infty$. В этом случае, как показано в работах автора [3] и [8], дифференциально-операторное уравнение вида (2.10), удовлетворяющее начальным условиям (2.6), имеет решение и оно представляется в следующем виде:

$$
u(t, z)=\sum_{k=0}^{m-1} u_{k}\left(t, z, f_{k}\right)+u_{r}(t, z, F)
$$

где

$$
\begin{gathered}
u_{k}\left(t, z, f_{k}\right)=\sum_{n=0}^{\infty} a_{n m+k} A^{n}\left(f_{k}\right) t^{n m+k}, \\
u_{r}(t, z, F)=\sum_{n=0}^{\infty} a_{m(n+1)} A^{n}(F) t^{m(n+1)}, \\
k=0,1, \ldots, m-1, \quad f_{0}, f_{1}, \ldots, f_{m-1}, F \in[1, \infty) .
\end{gathered}
$$

Как отмечалось выше (см. (2.4)), каждая функция $f_{0}, f_{1}, \ldots, f_{m-1}, F$ пространства $[\rho, \infty)$ представляется соответственно интегралом вида

$$
\begin{aligned}
f_{k}(z) & =\frac{1}{(2 \pi i)^{p}} \int_{\Delta r+\varepsilon} f(\xi \cdot z) \gamma_{f_{k}}(\xi) d \xi, \quad k=0, \ldots, m-1, \\
F(z) & =\frac{1}{(2 \pi i)^{p}} \int_{\Delta r+\varepsilon} f(\xi \cdot z) \gamma_{F}(\xi) d \xi
\end{aligned}
$$

Действуя на эти функции оператором $A$, находим

$$
\begin{aligned}
& A^{n}\left(f_{k}\right)(z)=\frac{1}{(2 \pi i)^{p}} \int_{\Delta r} e^{(\xi \cdot z)} \gamma_{f_{k}}(\xi) \varphi^{n}(\xi) d \xi, \quad n=0,1, \ldots \\
& A^{n}(F)(z)=\frac{1}{(2 \pi i)^{p}} \int_{\Delta r+\varepsilon} e^{(\xi \cdot z)} \gamma_{F}(\xi) \varphi^{n}(\xi) d \xi
\end{aligned}
$$

Подставляя эти выражения в (2.13), (2.12), получаем искомые представления (2.9), (2.8), (2.7).

Отметим, что абсолютная сходимость рядов $(2.12)$ и $(2.13)$ в $H\left(\mathbb{C}^{p}\right)$ (а следовательно, и рядов (2.8) и (2.9)), а также непрерывность решения (2.7) относительно начальных данных и неоднородного члена уравнения (2.5) обеспечивается тем фактом, что $\beta(A)=0$ (см. [3], [8]).

Далее, непосредственной подстановкой убеждаемся, что функция (2.7) удовлетворяет всем условиям теоремы и является искомым решением задачи (2.5), (2.6). Теорема доказана. 
Выделим два наиболее часто встречающиеся в практике случая $(m=1$ и $m=2)$ теоремы 3 и напишем их в интегральной форме.

ПреДЛОЖЕНИЕ 3. Пусть $m=1$, тогда задача (2.5), (2.6) имеет решение при любых $f_{0}, F \in\left[\rho_{1}, \infty\right)$, оно непрерывно относительно этих функций и представляется в виде

$$
(t, z)=\frac{1}{(2 \pi i)^{p}} \int_{\Delta r} \tilde{f}(\xi \cdot z)\left\{f(t \varphi(\xi)) \gamma_{0}(\xi)+\left[\frac{f(t \varphi(\xi))-1}{\varphi(\xi)}\right] \gamma_{F}(\xi)\right\} d \xi
$$

где $\gamma_{0}(\xi), \gamma_{p}(\xi)$ - обобщенные ассоциированные функции соответственно с $f_{0}$ и $F$ по функции $\tilde{f}, \Delta r-$ тор, на котором функции $\gamma_{0}$ и $\gamma_{F}$ аналитические и такой, что

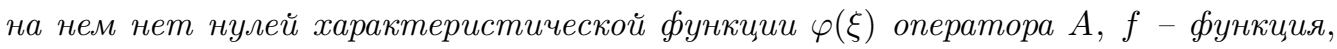
порождающая оператор $D_{f}$.

ПРЕДЛОЖЕНИЕ 4. Пусть $m=2$, тогда задача (2.5), (2.6) имеет решение при любых $f_{0}, f_{1}, F \in\left[\rho_{1}, \infty\right)$, оно непрерывно относительно этих функиий и представляется в следующем виде:

$$
\begin{aligned}
& u(t, z)=\frac{1}{(2 \pi i)^{p}} \int_{\Delta r} \tilde{f}(\xi \cdot z)\left\{\gamma_{0}(\xi) \operatorname{Kh}(t \sqrt{\varphi(\xi)})\right. \\
&\left.+\gamma_{1}(\xi) \frac{\operatorname{Sh}(t \sqrt{\varphi(\xi)})}{\sqrt{\varphi(\xi)}}+\gamma_{F}(\xi)\left[\frac{\operatorname{Kh}(t \sqrt{\varphi(\xi)})-1}{\varphi(\xi)}\right]\right\} d \xi
\end{aligned}
$$

где $\operatorname{Kh}(z)$ и $\operatorname{Sh}(z)$ - функиии, определенные в предложении 2.

В заключение отметим, что выражение

$$
\sum_{(k)} c_{(k)} D_{f}^{(k)}(F)(z) \equiv \varphi\left(D_{f}\right) F(z)
$$

можно рассматривать как обобщение свертки функционала, реализуемого последовательностью $\left\{c_{(n)}\right\}$, с функцией $F$ посредством оператора $D_{f}$. В частности, если $f(z)=e^{z_{1}} \cdots e^{z_{p}}$, то (2.14) представляет многомерную (классическую) свертку вида

$$
\sum_{(k)} c_{(k)} \frac{\partial^{(n)} F}{\partial z_{1}^{n_{1}} \cdots \partial z_{p}^{n_{p}}} \equiv \varphi\left(\frac{\partial}{\partial z}\right) F(z)
$$

Отметим также, что операторы вида

$$
\frac{\partial^{m}}{\partial t^{m}}-\varphi(D)
$$

широко распространены в различных разделах анализа. В частности, в упомянутой выше работе Стейнберга [5] рассмотрена задача Коши для дифференциально-операторных уравнений, содержащих оператор вида $(\partial / \partial t)-\varphi(d / d z)$, и доказана теорема существования и единственности решения в некоторых пространствах функций вещественного переменного. 


\section{СПИСОК ЦИТИРОВАННОЙ ЛИТЕРАТУРЫ}

[1] M. Lax, Fluctuation and Coherence Phenomena in Classical and Quantum Physics, Gordon and Breach, New York, 1968.

[2] В.П. Громов, "Аналитические решения дифференциально-операторных уравнений в локально выпуклых пространствах", Докл. РАН, 394:3 (2004), 305-308.

[3] В. П. Громов, "Операторный метод решения линейных уравнений", Ученые записки ОГУ, 3, Орел, 2002, 4-36.

[4] F. Trèves, Ovcyanikov Theorem and Hyperdifferential Operators, Notas de Matemática, 46, Instituto de Matemática Pura e Aplicada, Conselho Nacional de Pesquisas, Rio de Janeiro, 1968.

[5] S. Steinberg, "The Cauchy problem for differential equations of infinite order", J. Differential Equations, 9:3 (1971), 591-607.

[6] Л. В. Овсянников, "Сингулярный оператор в шкале банаховых пространств", Докл. AH CCCP, 163 (1965), 819-822.

[7] А. О. Гельфонд, А.Ф. Леонтьев, “Об одном обобщении ряда Фурье”, Матем. сб., 29 (71):3 (1951), 477-500.

[8] В.П. Громов, "Порядок и тип линейного оператора и целые векторнозначные функции", Ученые записки ОГУ, 1, Орел, 1999, 6-23.

[9] А.Ф. Леонтьев, Обобщения рядов экспонент, Наука, М., 1981.

[10] Л. Хёрмандер, Введение в теорию функиий нескольких комплексных переменных, Мир, М., 1968.

\section{В.П. Громов}

Орловский государственный университет
Поступило

19.10 .2005

Исправленный вариант

11.01 .2007 